EPJ Web of Conferences 66, 10013 (2014)

DOI: 10.1051/epjconf/ 20146610013

(C) Owned by the authors, published by EDP Sciences, 2014

\title{
Simulation toolkit with CMOS detector in the framework of hadrontherapy
}

\author{
R. Rescigno ${ }^{1, a}$, Ch. Finck ${ }^{1}$, D. Juliani ${ }^{1}$, J. Baudot ${ }^{1}$, D. Dauvergne ${ }^{2}$, G. Dedes ${ }^{2}$, J. Krimmer $^{2}$, C. \\ Ray $^{2}$, V. Reithinger ${ }^{2}$, M. Rousseau ${ }^{1}$, E. Testa ${ }^{2}$, and M. Winter ${ }^{1}$ \\ ${ }^{1}$ Institut Pluridisciplinaire Hubert Curien, F-67037 Strasbourg, France \\ ${ }^{2}$ Université de Lyon, Université Claude Bernard Lyon 1, CNRS/IN2P3, Institut de Physique Nucléaire de \\ Lyon, 69622 Villeurbanne, France
}

\begin{abstract}
Proton imaging can be seen as a powerful technique for on-line monitoring of ion range during carbon ion therapy irradiation. The protons detection technique uses, as three-dimensional tracking system, a set of CMOS sensor planes. A simulation toolkit based on GEANT4 and ROOT is presented including detector response and reconstruction algorithm.
\end{abstract}

\section{Introduction}

Hadrontherapy is a technique which makes use of charged particles (mainly protons and carbon ions) to treat local tumors. Compared to conventional radiotherapy, this technique presents two advantages: a precise ballistic with a finite range and a maximum dose deposition at the end of the path of the ions (called Bragg peak) and an enhanced biological efficiency in the Bragg peak region (mainly for carbon). The range of particles depends on their energy and on the electron density of material they are passing through, resulting in a steep dose gradient at the distal edge of the Bragg peak. Positioning of these dose gradients is critical for treatment planning and patient irradiation: an uncertainty of even few millimeters can lead to under-dosage in target volume or over-dosage of an organ at risk. However, there are several patient-related uncertanty sources influencing the particle range (inaccuracy arising from the $\mathrm{x}$-ray $\mathrm{CT}$ image used for the planning, patient mis-positioning, morphological changes in either tumor or healthy tissue volumes during the treatment period, organ motion), and requiring additional safety margins to ensure a complete irradiation of the tumor [1]. In order to reduce these margins and deliver a safer treatment, a device for the real-time monitoring of the range inside the patient would be useful. Several techniques have been explored for this purpose: PET monitoring which was used off line [3] and on line [2], the use of other two types of secondary particles emerging from patient, (i) prompt gamma [4][5] and charged particles, in particular protons (for carbon beams) [6][7]. The present paper focuses on proton imaging (IVI method) in the frame of the QAPIVI (Quality Assurance by Protons Vertex Imaging) project. The aims of the project is to finalize the study started in ref. [7], by simulation and experiments, including detector resolution and target inhomogeneities. For this purpose a simulation package based on GEANT4 [8] (version 4.9.5.1) and

\footnotetext{
ae-mail: regina.rescigno@iphc.cnrs.fr
} 
ROOT [9] has been developed. In this paper the detector simulation, reconstruction algortithms and first results obtained with an homogeneous target will be presented.

\section{The method}

The IVI (Interaction Vertex Imaging) method uses charged particles produced in the fragmentation of the incident ions to obtain information about the beam range. The principle is to reconstruct trajectories of emitted particles and to extrapolate them to the point of creation, called vertex. In the case of ion carbon therapy, it has been shown that a large number of protons are generated during collision along the primary ion path [10] and the multiplicity of protons was found to be enough to perform ion-range monitoring on a pencil-beam basis operating in active mode in homogeneous targets [7] .

\section{Simulation toolkit features}

The developed simulation package allows to reproduce the whole experimental setup composed of: (i) a beam monitoring to define the position and the direction of the beam; (ii) a target region (several geometrical forms and materials are possible): in this study an homogeneous parallelepiped PMMA target (density $1.19 \mathrm{~g} \mathrm{~cm}^{-3}$ ), $100 \mathrm{~mm}$ high, $100 \mathrm{~mm}$ wide and $250 \mathrm{~mm}$ thick has been used; (iii) a three-dimensional tracking system made of a set of thin silicon pixel detectors based on CMOS technique, to register the trajectory of secondary protons.

\subsection{The detector and beam monitoring}

The tracking system is made of four planes of two MIMOSA26 (M26) chips [11], each plane is separated by $2 \mathrm{~mm}$ in longitudinal direction, and the distance between the first plane and the target center is about $20 \mathrm{~cm}$. The M26 chip has a sensitive area of $10.6 \times 20.2 \mathrm{~mm}$ and is covered by 576 lines and 1152 columns of pixels with $18.4 \mu \mathrm{m}$ pitch. A pixel is considered as fired when the charge deposited is higher than a given threshold: the output is binary. The resolution of such a device for hadrontherapy purposes is better than $10 \mu \mathrm{m}$ [12].

When an ionizing particle passes through the M26 chip, the produced charge is collected by a given number of pixels. A group of hits generated by the same particle is called cluster. Figure 1 (left) shows the cluster size for a $80 \mathrm{MeV}$ protons beam and $80 \mathrm{MeV} /$ nucleon carbons beam (experimental data from FIRST experiment [12]). The two distributions are clearly separated: the protons one (black) is peaked at 4.4 pixels while the one for carbon ions is peaked at 21.2 pixels with a standard deviation of 1.7 pixels and 4.7 pixels respectively. To reproduce the CMOS response i.e. the number of fired pixels as a function of deposited energy, a phenomenological model was implemented. Information from the present set of experimental data are used to tune the parameters of the function reproducing the charge collection [13]. In Figure 1 (right) results from simulation are reported: for protons (black) a mean value of 4.8 pixels and a width of 1.5 pixels are obtained; the carbon ions distribution (red) is picked at 21.5 pixels with a width of 2.14 pixels. For both samples, the mean values of simulated distributions are in agreement with the experimental ones.

An additional pair of planes M26 chip are placed upstream the target and are used as beam monitoring and as trigger.

\subsection{Reconstruction algorithms}

The reconstruction chain is in three steps: first, the fired pixels are gathered to identify clusters on each plane (clustering); aligned clusters are searched to build a track (tracking) and, finally, reconstructed tracks are used to estimate the vertex position (vertexing). Vertex is identified as the middle 

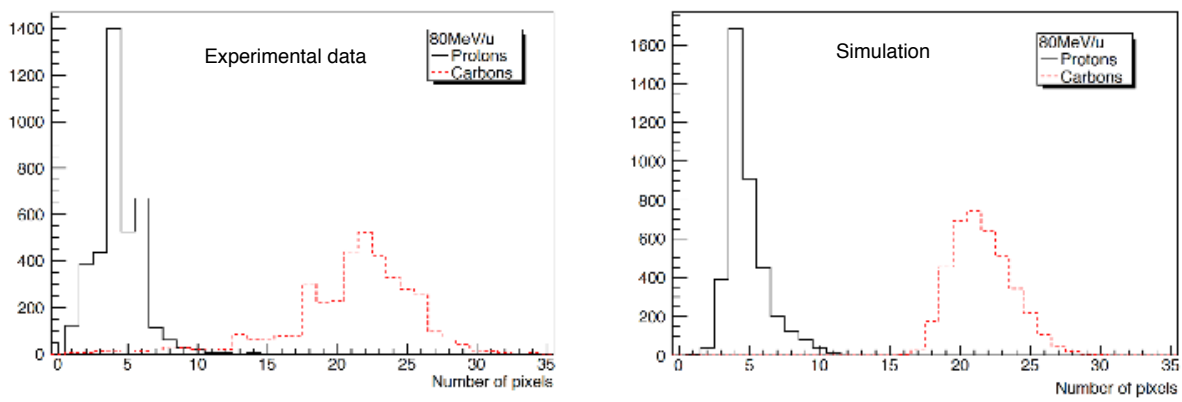

Figure 1. Number of pixels per cluster for protons (black) at $80 \mathrm{MeV}$ and carbons (red) at $80 \mathrm{MeV} / \mathrm{nucleon}$ : experimental (left) and simulated (right) data comparison.

point of the segment of minimal distance between incident trajectory and secondary proton tracks. The performance of reconstruction algorithms was evaluated by using a thin target $(\sim 8 \mathrm{~mm})$. An efficiency of about $99 \%$ was found both for tracking and vertexing algorithm. In particular, for vertexing algorithms, a resolution of about $11 \mu \mathrm{m}$ in $(X, Y)$ plane and less than $60 \mu \mathrm{m}$ in longitudinal direction is achieved [13].

\section{Results and discussion}

A simulation with $10^{6}$ carbon ions of $372 \mathrm{MeV} /$ nucleon was carried out, according to the number of incident ions necessary to perform a real-time ion range monitoring. At this energy and in a PMMA target, the carbon ion range is of about $210.4 \mathrm{~mm}$.

Recorded events have been processed and longitudinal vertex position was estimated. The true vertices profile (obtained using true vertex position) is compared with the reconstructed one in Figure 2 (left). The shape of the two distributions is slightly different: some vertices are reconstructed outside the target with a consequent deficit of vertices at target entrance and exit. This results does not depend on either detector, having a micrometric resolution, or tracking/vertexing algorithms, able to reconstruct tracks/vertices with an high efficiency. It's mainly the proton straggling in target that leads to an indetermination in the vertex position reconstruction. To quantify the vertices resolution, the residuals (i.e. difference between true and reconstructed vertex positions), are shown in Figure 2 (right). The resolution achieved in longitudinal direction is of $15 \mathrm{~mm}$. However, this spatial resolution is not the most critical issue of the IVI technique; the monitoring mainly relies on the information provided by the fall-off position. It has been shown in ref. [7] that a millimetric precision on the fall-off position of the vertex distribution can be achieved on a pencil-beam basis in homogeneous target.

\section{Conclusion}

Ion-range monitoring during carbon ion therapy by means of interaction vertex imaging (IVI) with secondary protons was investigated by using planes of MIMOSA26 chips as tracker device. A simulation package based on GEANT4 and ROOT was developed, including detector response and reconstruction algorithms. Detector features and algorithm performances allow to reach a vertices resolution of about $60 \mu \mathrm{m}$ for a $8 \mathrm{~mm}$ thick target and of about $15 \mathrm{~mm}$ for a $250 \mathrm{~mm}$ thick one. The main conclusion is that the estimation on vertex position is mainly affected by protons straggling in 

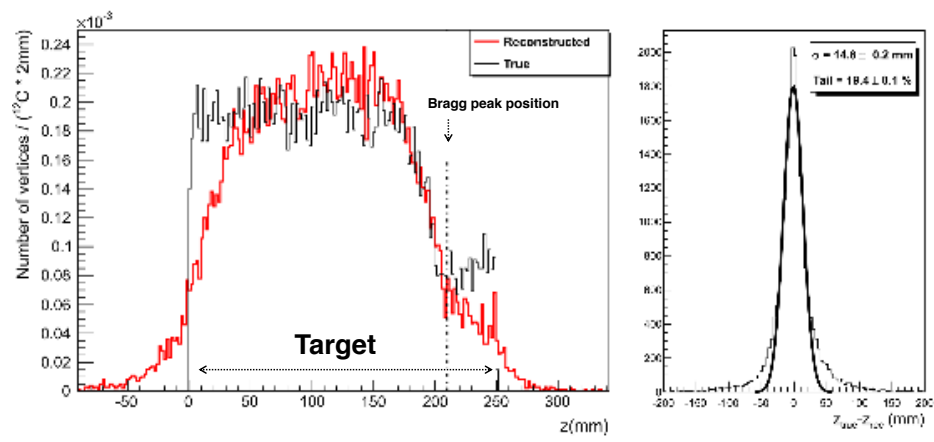

Figure 2. Left: True (black) and reconstructed (red) longitudinal vertices distribution for $372 \mathrm{MeV} / \mathrm{nucleon}$ carbon ions incident on a PMMA target; the estimated position of Bragg peak is shown with a black dash line. Right: Residual distance between simulated (true) and reconstructed (rec) vertices in longitudinal projection. Relative proportion of events outside the window $\pm 4 \sigma$ (tail) is reported.

the target and not by spatial resolution of the tracking system nor by the efficiency of reconstruction algorithms.

Experiment with homogeneous target have validated the IVI methods [14][15]. Measurements with heterogeneous targets have been performed at HIT (Heidelberg Ion-beam Therapy center) in April 2013: data analysis is in progress.

\section{Acknowledgment}

This work was partly supported by INCa (Physique Cancer projet QAPIVI), the ENVISION European project (grant agreement n241851), and was partly performed in the frame of the Labex PRIMES (ANR-11-LABX-0063)

\section{References}

[1] McGowan S. E. et. al., Br. J. Radiol. 8620120288 (2013)

[2] Enghardt W. et al., Nucl. Instrum. Methods A 525 284-8 (2004)

[3] Levy, R. P., Nucl. Instrum. Methods Phys. Res. B 261, 782-785 (2007).

[4] Testa E. et al., Applied Physics Letters 93093506 (2008)

[5] Min C-H. et al., Applied Physics Letters 89 183517-3 (2006)

[6] Amaldi U. et al., Nucl. Instrum. Meth. A 617 248-9 (2010)

[7] Henriquet P. et al., Phys.Med.Biol57 4655-69 (2012)

[8] Apostolakis J. et al., Radiat. Phys. Chem. 78 859-73 (2009)

[9] Brun R., Nucl.Instrum.Methods A 389 81-6 (1997)

[10] Gunzert-Marx K. et al.,New J. Phys. 10075003 (2008)

[11] Hu-Guo et al., Nucl. Instrum. Meth. A 623480 (2010)

[12] Spiriti E. et al., to be published

[13] Rescigno R. et al., to be published

[14] Gwosch K. et al., Phys.Med.Biol 58 3755-73 (2013)

[15] Reithinger V. et al., to be published 